rheuma plus $2019 \cdot 18: 1$

https://doi.org/10.1007/s12688-019-0243-1

(c) Springer-Verlag GmbH Austria, ein Teil von

Springer Nature 2019

\section{Burkhard Leeb}

Institut für Klinische Rheumatologie, Karl Landsteiner Gesellschaft, Hollabrunn, Österreich

\title{
Allgemeinmediziner und Rheumatologie
}

\section{Liebe Leserinnen und Leser!}

Ein neues Jahr hat begonnen, die Fragestellungen und zu lösenden Aufgaben sind die alten geblieben. Der Ruf nach individualisierter Betreuung wird immer lauter; die alten, sich an Gruppen orientierenden, Algorhythmen scheinen mehr und mehr ausgedient $\mathrm{zu}$ haben. Allerdings wird die individualisierte Betreuung deutlich mehr Rheumatologen benötigen als in Zeiten des Ärztemangels zur Verfügung stehen werden, auch wenn Politik und Verwaltung uns immer noch weismachen wollen, dass immer bessere Versorgung durch immer weniger Fachpersonal und selbstverständlich billiger bewerkstelligt werden können.

Immer schon und in zunehmendem Maße haben daher Allgemeinmediziner eine entscheidende Rolle in der Betreuung rheumatologischer Patienten. Als erste Anlaufstelle haben sie zu beantworten, ob Beschwerden entzündlicher oder nicht entzündlicher Natur sind. Im Fall von entzündlichen Beschwerden ist keine Zeit zu verlieren und die Patienten sollten möglichst rasch zum Rheumatologen überwiesen werden. Daher sollten sich die Allgemeinmediziner eher auf die Differenzierung entzündlich - nicht-entzündlich konzentrieren und weniger auf eine exakte Diagnosestellung.

Darüber hinaus erfolgt die alltägliche Betreuung rheumatologischer Patienten $\mathrm{zu}$ einem großen Teil in der Allgemeinpraxis, weil es einfach zu wenige Rheumatologen in der freien Praxis gibt, vor allem auch weil in der Kassenpraxis der ausschließlich als Rheumatologe tätige Arzt bei den von den Sozialversicherungen bezahlten Honoraren finanziell nicht überleben kann. Aus diesem Umstand ergibt sich die Notwendigkeit einer engen
Zusammenarbeit zwischen Rheumatologen und Allgemeinmedizinern und eines möglichst hohen Ausbildungsstandards der Kollegen in der Praxis, besonders hinsichtlich neuer Therapieoptionen und deren möglicher Komplikationen. Darüber hinaus sollten Hausärzten möglichst einfache Instrumente zur Krankheitsaktivitätsmessung zu Verfügung gestellt werden, so wie z. B. der RADAI-5 Fragebogen.

Zumeist werden in der Allgemeinpraxis Gichtpatienten betreut, wobei häufig vergessen wird, dass die Gicht die häufigste entzündliche Gelenkerkrankung überhaupt darstellt. Neben den häufigen rheumatologischen Krankheitsbildern gibt es aber auch selten solche, wo der Gedanke daran bereits die halbe Miete zum richtigen Management darstellt. Dazu zählt die Sarkoidose. Wie, wann und womit wir behandeln sollen beleuchtet Josef Hermann, Graz. Ebenfalls selten, aber doch immer wieder auftretend ist das Antiphospholipid-Syndrom; Christof Specker, Essen, beschreibt den State of the Art und neue Entwicklungen bei dieser teilweise lebensgefährlichen Erkrankung.

Im individualisierten Behandlungsplan spielen nicht nur medikamentöse Therapien, sondern auch komplementäre, balneologische und auch rehabilitative Maßnahmen eine große Rolle. Zum Teil als Ergänzung, zum Teil als Alternativtherapie, weil Patienten der klassischen Schulmedizin skeptisch gegenüberstehen. Daher erscheint das Wissen um die Möglichkeiten und die Grenzen dieser Therapieformen von enormer Wichtigkeit, um die Patienten wirklich umfassend beraten zu können. Alexander Meng, Wien, beschäftigt sich mit Traditioneller Chinesischer Medizin und Akupunktur, also Optionen aus zwei Welten.
Auch für die Rheumakinder wurden riesige Fortschritte erzielt, die allgemein etwas zu wenig Würdigung in der Gesamtrheumatologie finden. G. Horneff, Kinderrheumazentrum St. Augustin, gibt einen Überblick über entzündliche Gelenkerkrankungen im Kindesalter. Ein Fallbericht von Michale Borte, Leipzig, ergänzt dieses Paper in kongenialer Weise.

Wir hoffen, auch mit dieser Ausgabe wieder Ihr Interesse wecken $\mathrm{zu}$ können, um dem Ziel von rheuma plus nahe zu kommen, eine fruchtbringende, offene und redliche Diskussion in Gang zu bringen, aus der alle Nutzen ziehen können. Wie immer an dieser Stelle möchten wir Sie herzlich dazu einladen, uns Ihre Meinung zu den Beiträgen dieser Ausgabe, wie auch zu allen rheumatologischen Themen, die Sie für relevant oder für diskussionswert halten, zu schicken. Sie können in jedem Fall auf einen fairen Review mit entsprechendem Feedback vertrauen. Für Kritik, Hinweise, Zustimmung wie auch für jeden anderen Beitrag bedanke ich mich schon im Voraus.

Herzlichst,

\section{Ihr Burkhard Leeb}

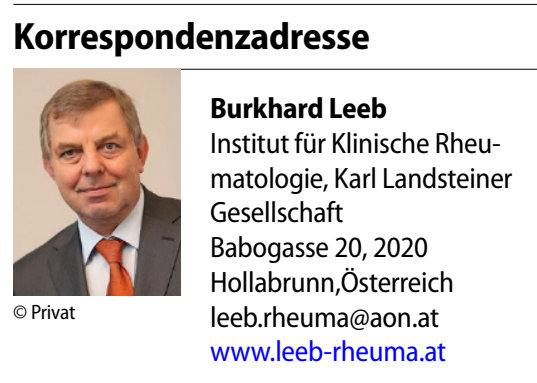

Interessenkonflikt. B. Leeb gibt an, dass kein Interessenkonflikt besteht. 